\title{
Neuroleptic Malignant Syndrome associated with the use of LAI antipsychotics: how to avoid?
}

\author{
Pedro, M.O.P. 1; Campos, S.B.F.1; Pedro, M.2; \\ Malinowski, F.R.L ${ }^{3}$ \\ maria.oliviapozzolo@gmail.com
}

$\underbrace{£}_{\text {FA G }}$ CENTRO $\quad \begin{gathered}{ }^{1} \text { Resident in Psychiatry FMABC } \\ \text { 2Medical Student at FAG University Center }\end{gathered}$

Fq fmabc

Disciplinas de Psiquiatria e Psicologia Médica da FMABC

\section{INTRODUCTION}

Neuroleptic malignant syndrome (NMS) is an uncommon but serious adverse effect in antipsychotics treatment, both oral and depot administration, and others. L-dopa drugs, antidepressants and antihistamines (1). The four main symptoms are hyperthermia, muscle stiffness, autonomic dysfunction and altered level of consciousness, may be seen still rhabdomyolysis and leukocytosis (2), being a diagnosis of exclusion. NMS is an important cause of morbidity and mortality (10\%) among patients taking antipsychotics. It occurs mainly at the beginning of treatment $(80 \%)$ and theprevalence varies from $0.07 \%$ to $2.4 \%$. The pathogenesis of NMS has been convincing in exacerbated dopamine receptor blockade (3). Other cofactors may be identified, such as norepinephrine system imbalance, GABA and serotonin. Central dysregulation of the adrenergic sympathetic system may also be a probable factor of the etiology $(4,5)$.

Paliperidone palmitate (9-hydroxy-risperidone) is a psychotropic agent belonging to is chemistry of benzisoxazole (atypical neuroleptic antipsychotic) and is an active metabolite of risperidone. Palideridone palmitate is hydrolyzed to paliperidone. A paliperidone is a centrally acting D2 dopaminergic antagonist with predominant serotonergic 5HT2A antagonist activity. Long-term (injectable) medication is effective 8 to 22 days after administration.

\section{REPORT}

P.A.S., 43 years old, female, psychiatric history, previously diagnosed with schizoaffective disorder, treated at an outpatient psychiatry clinic for 10 years and followed up at Vera Cruz Clinic for 1 month. It presented psychomotor agitation, logorrhea, disconnected and sometimes disorganized speech, impoverished, delusional and uncritical content. Patient had switched from oral to intramuscular medication two weeks before hospitalization, use of palipedirone $100 \mathrm{mg} / \mathrm{month}$. The change in drug administration route was due to poor adherence to treatment

During hospitalization, the patient evolves with muscle stiffness, difficulty walking and fine tremors, without the presence of a cogwheel, maintaining confused and disconnected thinking, with referred auditory hallucinations, complaining of reverberation of thoughts and logorrhea. After seven days of hospitalization, she began with changes in vital signs, hyperthermia $\left(38.5^{\circ} \mathrm{C}\right)$ and tachycardia, accompanied by muscle stiffness, poor contact, psychomotor agitation, with the presence of cogwheel, reported fetid urine, oliguria, sudoreic. In the first evaluation was suspected NMS and sepsis, where antibiotic and clinical support was introduced, antipsychotic withdrawn and referred for clinical evaluation. Patient is discharged after intensive care, taking benzodiazepines, and vital sign control is maintained. On the seventh day after return, aripiprazole was introduced with improvement of the psychopathological condition.

\section{Diagnostic Criteria for NMS}

1. Exposure to dopamine antagonist, or dopamine agonist withdrawal, within past 72 hours.

2. Hyperthermia $\left(>100,4^{\circ} \mathrm{F}\right.$ or $>38.0^{\circ} \mathrm{C}$ on at least 2 ocasions, measured orally.

3. Rigidity.

4. Mental status alteration (reduced or fluctuating level of consciousness).

5. CK elevation (at least 4 times upper limit of normal)

6. Sympathetic nervous system lability, defined as at least 2 of the following:

a. Blood pressure elevation (systolic change or $\geq 25 \%$ above baseline)

b. Blood pressure fluctuation $(\geq 20 \mathrm{mmHg}$ diastolic change or $\geq$ $25 \mathrm{mmHg}$ systolic change within 24 hours)

c. Diaphoresis

d. Urinary incontinence

7. Hypermetabolism, definied as heart rate increase $(>25 \%$ above baseline) and respiratory rate increase $(>50 \%$ above baseline)

8. Negative workup for infectious, toxic metabolic, and neurologic causes

\section{CONCLUSION}

The case presented in this report demonstrates a probable adverse reaction to the use of long-term depot paliperidone, an atypical antipsychotic. New insights into deposit medications should be put into practice. The use of depot paliperidone should be questioned in patients diagnosed with refractory schizoaffective disorder, being a very valid option for patients with poor adherence to oral medications. Neuroleptic malignant syndrome is a clinical condition that, although uncommon, is often present in the use of antipsychotics and other drugs, and early diagnosis and appropriate supportive treatment are of paramount importance. Concomitantly, the scientific literature lacks similar cases.

REFERENCES:

1. Ananth J, Aduri K, Parameswaran S, et al. Neuroleptic malignant syndrome: risk factors, pathophysiology, and treatment. Acta Neu- ropsychiatrica. 2004; 16(4):219-28.

Gurrera RJ, Chang SS, Romero JA. A comparison of diagnostic criteria for neuroleptic malignant syndrome. J Clin Psychiatry. 1992; 53:56-62.

Delay J, Pichot P, Lemperiere T. Un neuroleptique majeur non phenothiazine et non reserpinique l'haloperidol dans le traitment des psychoses. Ann Med Psychol, Paris. 1960; 118:145-152. Mann SC, Caroff SN, Fricchione G, Campbell C. Central dopamine hypoactivity and the pathogenesis of neuroleptic malignant syndrome. Psychiatr. Ann. 2000; 30(5), 363-374. . Gurrera RJ. Sympathoadrenal hyperactivity and the etiology of neuroleptic malignant syndrome. Am. J.

Psychiatry . 1999; 156 (2), 169-180.

6. Tiihonen J, Haukka J, Taylor M, Haddad PM, Patel MX, Korhonen P. A nationwide cohort study of oral and depot antipsychotics after first hospitalization for schizophrenia. Am J Psychiatry 2011;168:603- 609

American Psychiatric Association. Diagnostic and Statistical Manual of Mental Disorders. 5th ed. Arlington, VA: American Psychiatric Association; 2013 